\title{
HPV infections in adolescents
}

\author{
Anna-Barbara Moscicki* \\ Division of Adolescent Medicine, University of California, San Francisco, CA, USA
}

\begin{abstract}
Adolescents who are sexually active have the highest rates of prevalent and incident HPV infection rates with over $50-80 \%$ having infections within 2-3 years of initiating intercourse. These high rates reflect sexual behavior and biologic vulnerability. Most infections are transient in nature and cause no cytologic abnormality. However, a small number of adolescents will not clear the infection. Persistence of HPV is strongly linked to the development of high-grade squamous intra-epithelial lesions (HSIL) and invasive cancer. The HSIL detected, however, does not appear to progress rapidly to invasive cancer. Understanding the natural history of HPV in adolescents has shed light into optional treatment strategies which include watchful observation of atypical squamous cells of undetermined significance (ASCUS) and low grade (LSIL). The association between age of first intercourse and invasive cancer cannot be ignored. Consequently, initiating screening at appropriate times in this vulnerable group is essential. In addition, with the advent of the HPV vaccine, vaccination prior to the onset of sexual activity is critical since most infections occur within a short time frame post initiation.
\end{abstract}

Keywords: Natural history, HPV, SIL, adolescents

\section{Introduction}

One of the primary interests in human papillomavirus (HPV) is tied to its unique oncogenic properties. Virtually all squamous cell invasive cancers of the cervix are due to HPV infections. This striking finding is overshadowed by the commonness of this virus. HPV is the most common sexually transmitted infection with over $80 \%$ of sexually active women having been exposed to the virus; most within 3-4 years after sexual debut [1,2]. What still intrigues scientists is the long gap between first infection (usually under 21 years of age) and development of invasive cancer in women several decades later. The high prevalence of HPV in young women underscore the vulnerability of adolescents to HPV but the low prevalence of cervical cancer in this age group underscore the benign nature of initial HPV infections. The high incidence of HPV in young women plus the risk of invasive cancer and young age at first intercourse suggests that there are behavioral

* Address for correspondence: Anna-Barbara Moscicki, MD, University of California San Francisco, 3333 California Street, Suite 245, San Francisco, CA 94118, USA. Tel.: +1 415476 5139; Fax: +1 415476 6106; E-mail: moscickia@peds.ucsf.edu. and/or biologic risks that place the adolescent vulnerable to not only HPV but persistent HPV infection. This Chapter will discuss rates of acquisition in adolescents, the natural history of infections and dysplasia, biologic changes unique to adolescents and preferred treatment recommendations for HPV in adolescents.

\section{Incidence of HPV infection in adolescents}

Most prevalence studies have demonstrated a 6-8 fold difference in HPV in younger women compared to older. Rates have ranged from $12 \%$ to $56 \%$ in women under 21 years compared to $2-7 \%$ in women over 35 years of age [3-6]. Although this trend has been relatively consistent in studies from developed countries, some countries show no increase across ages or a second increase in older women. A study from Costa Rica suggested that a second rise occurs after the age of 50 years; however, those rates still do not reach those found in adolescents [7].

In a study reported in 2001, approximately $55 \%$ of adolescents and young women acquired a cervical HPV infection within 36 months after joining the study [8]. This was approximately within 5-7 years after initiating sexual intercourse. The greatest risk factor for hav- 
ing an incident infection was reporting a new sexual partner. The risk was over 10 fold for each new partner reported per month since the last visit 4 months ago. A recent analysis of a similar cohort showed that of 620 adolescent and young women entered into the study, $37 \%$ had a prevalent cervical infection at baseline and $63.3 \%$ had an incident infection with a cumulative rate of 75\% within approximately 5-6 years after sexual debut (unpublished; personal communication Moscicki).

Another recent study examined 60 adolescents who were asked to obtain weekly vaginal samples and had cervical samples obtained at 3-month intervals [2]. Seventy seven percent were found to have a positive sample for high risk HPV by 2.2 years of follow-up. The most common type in this study was HPV 52; $38.3 \%$ had this type detected followed by HPV 16 with a cumulative prevalence of $31.3 \%$. If only cervical infections were considered, $36 \%$ had a HR HPV type detected within follow-up showing that half of the detected HPV infections were from vaginal swabs only. This is not surprising since many more samples from the vagina were obtained in this study than from the cervix. On the other hand, this finding also suggests that many of the vaginal infections do not cause cervical infections and are not at risk for causing invasive cervical cancer.

Age related incidence rates, however, have not been consistent across all HPV types. In a recent study in Costa Rica, women under 25 years of age were also more likely to have infection with certain HPV types but not all. It was noted that the alpha nine HPV types (16 and 16 related types) were $2-3$ times more common in women under 25 years than women over 35 years [9]. However other types, such as type 61 or 71 increased with increasing age. Of note, these HPV types (61 and 71) are not high-risk types suggesting their presence is unimportant.

\section{Vulnerability}

The high incidence of HPV in adolescents has been frequently blamed on sexual behavior. Adolescents are much more likely than older women to have had multiple partners within a shorter time frame, such as 6-12 months, than older women. The ease of HPV transmission during sexual activity has been demonstrated in numerous studies $[8,10]$. But in addition, to high incidence rates, studies have shown that young age at first intercourse is a risk for the development of cancer in later life with a 3-4 fold increased risk of developing

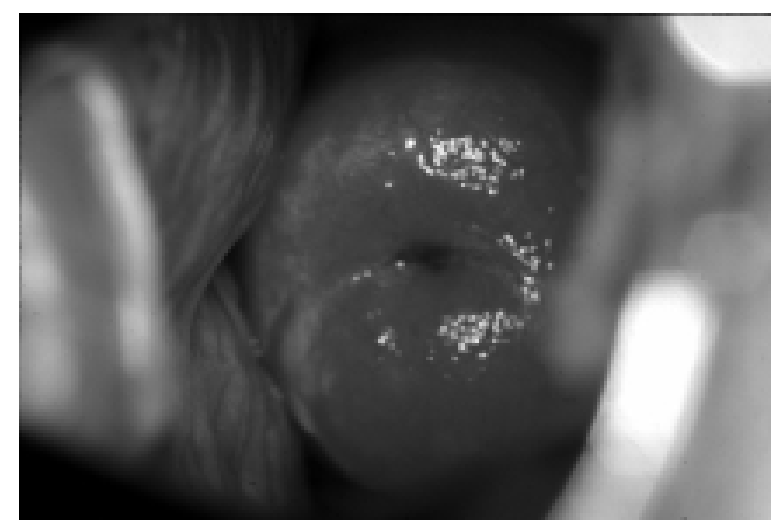

Fig. 1. Colpophotograph of a 16 year old. Note the large area of columnar epithelium in comparison to mature squamous epithelium and abrupt squamo-columanr junction seen well onto the ectocervix.

invasive cancer if a women initiated intercourse under the age of 18 years of age compared to 20 or older [ 11 , 12]. These studies imply that there may be a biologic vulnerability related to the cervix of young women. Certainly, the adolescent cervix in general is structurally different than the adult women's cervix [13]. Embryologically, the cervix is initially lined by columnar epithelium in utero. Urogenital squamous epithelium replaces the columnar epithelium; however the replacement is incomplete with an abrupt squamous-columnar junction occurring on the ectocervix. The adolescent enters puberty with relatively large areas of columnar epithelium (Fig. 1) compared to the adult women. Due to increased estrogenic activity and resulting acidity of the vagina, uncommitted generative cells of the columnar epithelium begin to transform themselves into squamous epithelium. Eventually, columnar epithelium transforms into squamous epithelium with a new columnar junction occurring well into the os. The predominant cell type in adults is the mature squamous cell (Fig. 2). Both columnar and metaplastic squamous cells are vulnerable to HPV probably for a variety of reasons of which thinness of the epithelium may be one factor (i.e. easy access to basal epithelial cells).

The transformation process is referred to as squamous metaplasia. The importance of the transformation zone is known since all squamous cell cancers of the cervix appear to arise within the transformation zone. Hence its vulnerability has been well established. Since the process of squamous metaplasia appears to be most active during adolescence, it may represent a vulnerability to the establishment of HPV infections. Triggers of squamous metaplasia include sexual activity. Adolescents with exposure to multiple sexual partners have cervixes that appear more adult 
Table 1

Cytologic categories using Bethesda

\begin{tabular}{ll}
\hline Bethesda Classification & W.H.O. Classification \\
\hline ASCUS (ASC) & Atypia \\
undetermined significance (ASC-US) & \\
cannot exclude HSIL (ASC-H) & \\
LSIL (low-grade squamous intra-epithelial lesions) & Condyloma \\
& CIN 1 \\
HSIL (high-grade squamous intra-epithelial lesions) & CIN 2, CIN 3. Carcinoma in situ \\
\hline
\end{tabular}

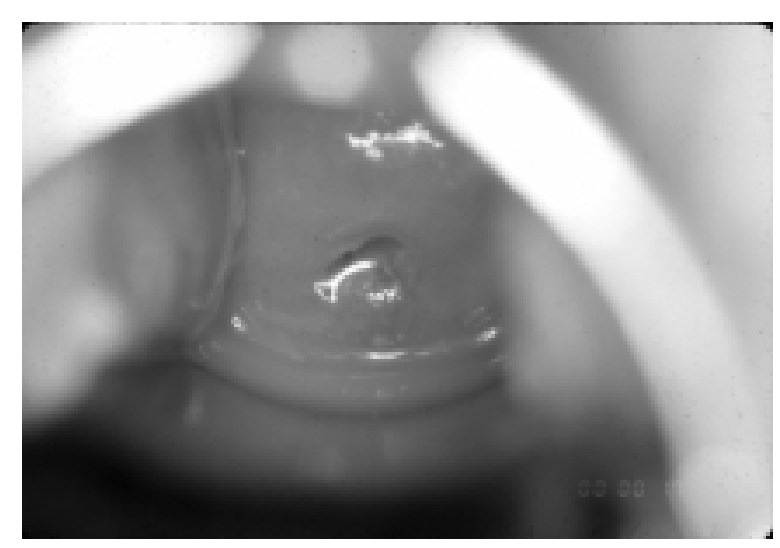

Fig. 2. Colpophotograph of a 20 year old. Note the entire cervix is covered by mature squamous epithelium with the squamo columnar junction not visible.

like with small areas of ectopy $[14,15]$. It may be women with multiple partners are exposed to sexually transmitted infections, which induce inflammation, repair and metaplasia or semen itself may induce metaplasia. Whatever the reason, rapidly proliferating cellular populations of squamous metaplasia is presumably vulnerable to HPV infection. HPV replication and patterns of transcription are highly dependent on the differentiation program of keratinocytes in the cervical squamous epithelium. Therefore, it is somewhat intuitive that squamous metaplasia is particularly favorable to HPV survival. The environment is conducive to viral replication, and possibly persistence. The high rates of LSIL (see Table 1 for definition of LSIL) in adolescent populations described below support this premise. The protective barrier reflected by the mature squamous epithelium may be an important factor why adult women have lower rates of infection. Rates of LSIL range from 2 to $14 \%$ [16-19] in adolescents while in older women ( $>30$ years) the rates range from 0.6 to $1 \%$. It is important to emphasize, however, that HPV detection in adolescents is most commonly associated with normal cytology. Over three-quarters of HPV-infected adolescents have normal cytology [8]. Whether microscopic abnormalities that remain undetected occur in this group is still controversial. But either way, LSIL, particularly in adolescents appear as benign as HPV infections with normal cytology (see Natural histories of HPV, LSIL, and HSIL in adolescents below).

The expression of viral proteins result in basal cell proliferation, nuclear enlargment and abnormal mitotic figures; all features of SIL [20]. A study of adolescent women showed that those large amounts of metaplastic change visible on colpopophotographs were more likely to develop LSIL if infected with HPV than adolescents with a relatively quiescent cervixes [21].

Few studies have attempted to examine biologic vulnerability to HPV infection. Castle et al. [22] found that the alpha nine types $(16,31,33,35,52,58$, and $67)$ were detected more commonly among women with greater areas of ectopy. However, the opposite was true of the $3 \alpha 15 \alpha$ types $(61,71,72,81,83,84,89)$ where prevalence increased with increasing maturity. These authors suggested that the epithelial type helped explain their findings.

\section{Natural histories of HPV, LSIL and HSIL in adolescents}

Numerous studies have documented the transient nature of HPV infection in adolescents and young women with $50 \%$ of women clearing an initially detected infection within 6 months, and 90\% clearing within 24 months $[1,23,24]$. It appears that certain HPV types, such as HPV 16, clear more slowly than other high or low risk types. Certainly, new infections of the same type may occur blurring the true regression rates. There is also evidence that having multiple types of HPVs also slows clearance in adolescents [25,26]. Whether this reflects a global defect in the immune response or whether multiple HPV types act synergistically is unclear. These studies are also difficult to interpret since the reproducabilty between laboratories of detecting multiple types is low. C. trachomatis, a common infection in adolescents, has been shown by one study to enhance HPV persistence [27,28]. Since TH1 responses are thought to be responsible for HPV clearance, 
the authors hypothesized that $C$. trachomatis in some women may be associated with a Th2 type response which in turns results in HPV persistence. This finding with $\mathrm{C}$. trachomatis has not been seen in other studies of HPV persistence [29].

Certainly, many of the HPV infections result in LSIL as mentioned above. The benign nature of LSIL is demonstrated by its high rate of regression that parallels the high rate of regression of HPV. A study of adolescents and young women showed that $92 \%$ of LSIL regressed within 36 months of observation [26]. Only $3 \%$ of the adolescent women went onto develop HSIL. Rates of regression of both HPV and LSIL among older women appear to be less frequent; hence detection of HPV or LSIL in older women (defined as $>30$ years) most likely reflects an already persistent infection and an increased risk for HSIL.

The role of HPV persistence in the development of HSIL has been established. However, how long persistence is required for HSIL development remains controversial. One of the problems in defining such risks is that CIN 2 and 3 make up the term HSIL (see Table 1) and the natural history of CIN 2 and 3 are likely different. So studies using HSIL have found different rates than those using CIN 2 or 3 . Several countries that have collected rates for CIN 3 show that rates of CIN 3 appear to peak in women aged $27-30$ years; $7-10$ years after peak rate for HPV infections [30]. In comparison, the risk of developing cytologic "HSIL" in adolescents is probably equal that of women aged 20-30 years of age. Mount and colleagues [19] review of cytology found $0.7 \%$ of $15-19$ year olds had HSIL compared to $0.8 \%$ of women aged $20-29$ years and $0.7 \%$ in $30-39$ year-olds. In a nationwide organized cervical screening program in Norway, $0.2 \%$ of 20,000 smears from adolescents aged 15-19 years were reported as having HSIL [17]. The reason for these differences is that the rates of CIN 2 most likely out number the rates of CIN 3 in adolescents whereas in older women CIN 3 predominates. So these studies that detect HSIL cytology in adolescents most likely reflect CIN 2 cases.

Given these limitations, it appears that CIN 1 and 2 develop shortly after infection in some women with others taking up to 1-3 years before detection. Moscicki et al. [8] found that $25 \%$ of women developed LSIL within 3 years with the risk of of developing cytology diminishing after 3 years. Woodman et al. [31] noted that $3 \%$ of HPV negative and $7 \%$ of HPV positive adolescents developed HSIL within 19 months of acquiring HPV.

In general, the natural history of LSIL in adolescents parallels that of HPV with few progressing to HSIL. In a longitudinal study of LSIL, only $3 \%$ of adolescents went onto HSIL within 3 years [26]. In contrast, a retrospective chart review of adolescents $<19$ years of age with cytologic LSIL reported that $31 \%$ progressed to HSIL by 36 months [32]. Since this study involved chart reviews, it is not clear if the HSIL reflected new lesions or actual progression of LSIL. In addition, only a third of the original cohort was followed for 36 months. These differences between studies may be a matter of interpretation of cytology and histology or a reflection of different population characteristics. None of the studies found invasive cancer. In comparison to older women, Cox et al. [33] showed that $12.8 \%$ of older women will progress from LSIL or ASCUS/HPV positive to HSIL within 2 years.

Studies of HSIL are few since carcinoma-in-situ is part of what is now termed HSIL and there are ethical concerns in monitoring these lesions without treatment. In addition,

CIN 2 is not a very reproducible diagnosis by pathologists and there is considerable debate as to whether CIN 2 behaves more like CIN 1 or more like CIN 3 [34]. The importance of these differences for adolescents is that CIN 2 lesions make up the majority of HSIL while CIN 3 is less common [16,19]. No studies to date are available for progression of CIN 2 or 3 in adolescents. However, prevalence studies in US adolescents have consistently shown that adolescents rarely have invasive cancer $[16,19]$. According to the most recent US SEER statistics (1995-1999), the incidence of invasive cancer in women under among 20 years of age is 0 3 per million [35]. Little information is available on the rare cases of invasive cancer such as presence of immunodeficiency disorders.

\section{Cervical cancer screening}

The high rates of regression in adolescents of HPV and LSIL have spurred a movement in the US to push back the age when to initiate screening. Older guidelines included all adolescents once sexually active [36, 37]. Recently, guidelines were constructed on risk behaviors (i.e. initiating sexual activity) and a time frame [38]. Through cost effective modeling, it was shown that initiating screening 3 years after the onset of sexual activity or 21 years of age, whichever comes first, would be cost-effective. In addition, these guidelines were felt to encompass screening in highrisk groups (i.e. those initiating sexual activity at early ages) $[36,37]$. 
Although cervical cancer screening in adolescents yield low benefits, the age limit to begin screening remains controversial. As mentioned, US guidelines base risk of cancer on sexual behavior. This type of screening is strategic in identifying the highest risk youth. Although the recommendations were based on sexual behavior reporting, most agree that a cap is needed for women who are unwilling or unable to report sexual activity. The upper age limit of 21 years used by the American Cancer society was primarily based on expert opinion. In the US, this age was considered a realistic age for compliance and access to patients, particularly that the US has no organized screening program. In countries with organized screening such as the United Kingdom, new recommendations start screening at 25 years [39]. In contrast, Australia has new guidelines that recommend screening begin at the ages of 18 years, or one to two years after first sexual intercourse, whichever is later [40]. Data show that some rare cases of invasive cancer will occur in the 20-24 year old group; hence precancers in this group would be missed if screening begins after 20 years of age.

\section{ASCUS and LSIL triage}

The high rates of LSIL and HPV regression have led to more reasonable guidelines for adolescents [41]. ASCUS triage with HPV testing has been incorporated into many triage schemes. However, recent study of population performances show that up to $77 \%$ of adolescents with ASCUS will be positive for high-risk HPV types. This has called into question the use of HPV triage in adolescent populations [42]. Repeat cytology 6 to 12 months is now often favored over HPV triage. Similar conservative observation of LSIL by cytology is also recommended with repeat cytology 6 to 12 months later rather than immediate referral to colposcopy [43]. Since ASCUS-HR HPV type has a similar history as LSIL, similar strategies can be given to these lesions if HPV testing is performed on the ASCUS smear. At first repeat cytology, HSIL or higher should be referred for colposcopy. At second repeat cytology, any abnormality on repeat cytology for ASCUS, ASCUS HR, and LSIL follow-up leads to referral to colposcopy. Because of the high rates of HPV infection in adolescents, follow-up with HPV testing for any of these lesions is of questionable value. No cost-effective analysis for this age group has been performed.

Appearance of HSIL on cytology at any follow-up visit should be referred to colposcopy.
Because of the frequency of HPV infections in adolescents, HPV testing for primary screening is not recommended.

\section{Treatment of SIL}

Treatment of LSIL among adolescents is considered unwarranted. Recommendation for treatment of HSIL for adolescents is similar to that of adults. Some have recommended cryotherapy over LEEP for suitable cases (small accessible lesions) in adolescents because of the lower rate of complication associated with cryotherapy. A recent meta-analysis of LEEP procedures showed increased risk of preterm delivery, low birth weight, and premature rupture of membranes [44].

In summary, HPV is undoubtedly the cause of cervical cancer. However, HPV detection, specifically in adolescents, is not an absolute marker of cervical cancer risk. Adolescents are at high risk of acquiring HPV infection with over 50-80\% having infections within 2-3 years of initiating intercourse. These high rates are due either to sexual behavior, biologic vulnerability or a combination of both. Most infections are transient in nature and cause no cytologic abnormality. However, a small number of adolescents will not clear the infection and develop HSIL. The HSIL detected, however, does not appear to progress rapidly to invasive cancer since cancers in this age group remain rare. Understanding the natural history of HPV in adolescents has shed light into optional treatment strategies which include watchful observation of ASCUS and LSIL. The association between age of first intercourse and invasive cancer cannot be ignored. Consequently, initiating screening at appropriate times in this vulnerable group is essential. With the HPV vaccine on the horizon, it will be essential to vaccinate children prior to the onset of sexual activity; prior to acquisition of HPV. Accordingly, adolescents who initiate sexual activity may not have yet developed an HPV infection and should receive vaccination as well. All adolescents vaccinated must be counseled about the important of continued cancer screening.

\section{References}

[1] A.B. Moscicki, S. Shiboski, J. Broering, K. Powell, L. Clayton, N. Jay, T.M. Darragh, R. Brescia, S. Kanowitz, S.B. Miller, J. Stone, E. Hanson and J. Palefsky, J Pediatr 132 (1998), 277.

[2] F.M. Brown, W.C. Faquin, D. Sun, C.P. Crum and E.S. Cibas, Am J Clin Pathol 112 (1999), 765. 
[3] A.B. Moscicki, J. Palefsky, J. Gonzales and G. Schoolnik, Pediatr Res 28 (1990), 507.

[4] A.B. Moscicki, J.H. Ellenberg, S.H. Vermund, C.A. Holland, T. Darragh, P.A. Crowley-Nowick, L. Levin and C.M. Wilson, Arch Ped Adolesc Med 154 (2000), 127.

[5] C. Ferreccio, R.B. Prado, A.V. Luzoro, S.L. Ampuero, P.J. Snijders, C.J. Meijer, S.V. Vaccarella, A.T. Jara, K.I. Puschel, S.C. Robles, R. Herrero, S.F. Franceschi and J.M. Ojeda, Cancer Epidemiol Biomarkers Prev 13 (2004), 2271.

[6] W.D. Rosenfeld, S.H. Vermund, S.J. Wentz et al., Am J Dis Child 143 (1989), 1443.

[7] R. Herrero, A. Hildesheim, C. Bratti, M.E. Sherman, M. Hutchinson, J. Morales, I. Balmaceda, M.D. Greenberg, M. Alfaro, R.D. Burk, S. Wacholder, M. Plummer and M. Schiffman, J Natl Cancer Inst 92 (2000), 464.

[8] A.B. Moscicki, N. Hills, S. Shiboski, K. Powell, N. Jay, E. Hanson, S. Miller, L. Clayton, S. Farhat, J. Broering, T. Darragh and J. Palefsky, JAMA 285 (2001), 2995.

[9] P.E. Castle, A.C. Rodriguez, F.P. Bowman, R. Herrero, M. Schiffman, M.C. Bratti, L.A. Morera, D. Schust, P. CrowleyNowick and A. Hildesheim, Clin Diagn Lab Immunol 11 (2004), 399.

[10] A. Burchell, R. Winer, S. de Sanjose and E. Franco, Vaccine Monographs (2006), in press.

[11] J. Green, A. Berrington de Gonzalez, S. Sweetland, V. Beral, C. Chilvers, B. Crossley, J. Deacon, C. Hermon, P. Jha, D. Mant, J. Peto, M. Pike and M.P. Vessey, Br J Cancer 89 (2003), 2078.

[12] C.H. Sierra-Torres, S.K. Tyring and W.W. Au, Int J Gynecol Cancer 13 (2003), 617.

[13] A. Singer, in: The Cervix, J.A. Gordon and A. Singer, eds, WB Saunders, Philadelphia, 1978, p. 87.

[14] A. Singer, Br J Obstet Gynaecol 82 (1975), 81.

[15] A.B. Moscicki, Y. Ma, C. Holland and S.H. Vermund, J Infect Dis 183 (2001), 865.

[16] S.B. Sadeghi, E.W. Hsieh and S.W. Gunn, Am J Obstet Gynecol 148 (1984), 726

[17] T. Bjorge, A.B. Gunbjorud, F. Langmark, G.B. Skare and S.O. Thoresen, Cancer Detection and Prevention 18 (1994), 463.

[18] L.T.M. Schydlower, M.H. Greenberg and C.P.H. Patterson, Clinical Pediatrics 20 (1981), 723.

[19] S.L. Mount and J.L. Papillo, Pediatrics 103 (1999), 539

[20] D. Solomon, D. Davey, R. Kurman, A. Moriarty, D. O'Connor, M. Prey, S. Raab, M. Sherman, D. Wilbur, T.J. Wright and N. Young, JAMA 287 (2002), 2114.

[21] A.B. Moscicki, V. Grubbs-Burt, S. Kanowitz, T. Darragh and S. Shiboski, Cancer 85 (1999), 1139.

[22] P.E. Castle, J. Jeronimo, M. Schiffman, R. Herrero, A.C. Rodriguez, M.C. Bratti, A. Hildesheim, S. Wacholder, L.R. Long, L. Neve, R. Pfeiffer and R.D. Burk, Cancer Res 66 (2006), 1218.
[23] G.Y. Ho, R. Bierman, L. Beardsley, C.J. Chang and R.D. Burk, NEJM 338 (1998), 423.

[24] M. Evander, K. Edlund, A. Gustaffson, M. Jonsson, R. Karrlson, E. Rylander and G. Wadell, The Journal of Infectious Diseases 171 (1995), 1026.

[25] A.B. Moscicki, J.H. Ellenberg, S. Farhat and J. Xu, J Infect Dis 190 (2004), 37.

[26] A.B. Moscicki, S. Shiboski, N.K. Hills, K.J. Powell, N. Jay, E.N. Hanson, S. Miller, L. Canjura-Clayton, S. Farhat, J.M. Broering and T.M. Darragh, Lancet 364 (2004), 1678.

[27] I. Silins, W. Ryd, A. Strand, G. Wadell, S. Tornberg, B.G. Hansson, X. Wang, L. Arnheim, V. Dahl, D. Bremell, K. Persson, J. Dillner and E. Rylander, Int J Cancer 116 (2005), 110.

[28] E. Samoff, E.H. Koumans, L.E. Markowitz, M. Sternberg, M.K. Sawyer, D. Swan, J.R. Papp, C.M. Black and E.R. Unger, Am J Epidemiol 162 (2005), 668.

[29] A.B. Moscicki, J.H. Ellenberg, S. Fahrat and J. Xu, Journal of Infectious Diseases 190 (2004), 37.

[30] M. Schiffman and S.K. Kjaer, J Natl Cancer Inst Monogr 31 (2003), 14.

[31] C.B. Woodman, S. Collins, H. Winter, A. Bailey, J. Ellis, P. P., M. Yates, R.T. P. and L.S. Young, Lancet 357 (2001), 1831.

[32] J.D. Wright, R.M. Davila, K.R. Pinto, D.F. Merritt, R.K. Gibb, J.S. Rader, D.G. Mutch, F. Gao and M.A. Powell, Obstet Gynecol 106 (2005), 115

[33] J.T. Cox, M. Schiffman and D. Solomon, ASCUS-LSIL Triage Study (ALTS) Group, Am J Obstet Gynecol 188 (2003), 1406.

[34] K. Syrjanen, V. Kataja, Yliskoski, F. Chang and S. Syrjanen, Obstet Gynecol 79 (1992), 675.

[35] P.G. Chan, H.Y. Sung and G.F. Sawaya, Obstet Gynecol 102 (2003), 765.

[36] American College of Obstetricians and Gynecologists, 152 (1995).

[37] American Medical Association, in, American Medical Association, Chicago, IL, 1992.

[38] D. Saslow, C.D. Runowicz, D. Solomon, A.B. Moscicki, R.A. Smith, H.J. Eyre and C. Cohen, CA Cancer J Clin 52 (2002), 342.

[39] NHS Cancer Screening Programmes, in, NHS Cervical Screening Programme, http://www.cancerscreening.nhs.uk/ cervical/index.html\#invited, 2006.

[40] National Health and Medical Research Council, in, 2005.

[41] T.C.J. Wright, J.T. Cox, L.S. Massad, L.B. Twiggs, E.J. Wilkinson, JAMA 287 (2002), 2120.

[42] G. Sawaya, JAMA 294 (2005), 2210.

[43] American Society for Colposcopy and Cervical Pathology, in, 2006.

[44] M. Kyrgiou, G. Koliopoulos, P. Martin-Hirsch, M. Arbyn, W. Prendiville and E. Paraskevaidis, Lancet 367 (2006), 489. 


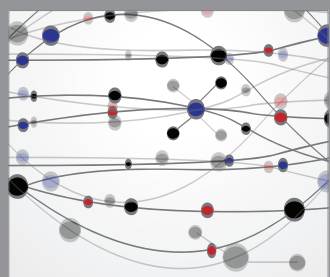

The Scientific World Journal
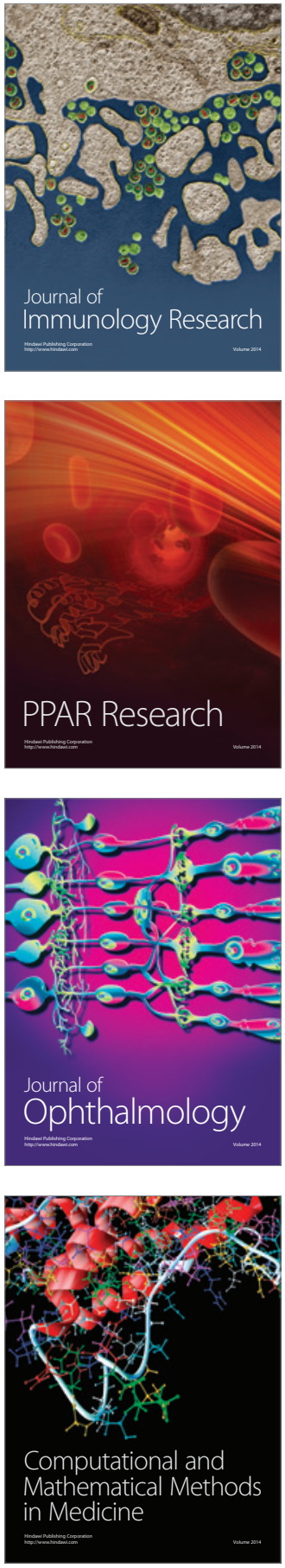

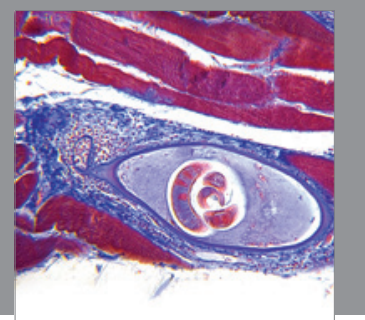

Gastroenterology

Research and Practice
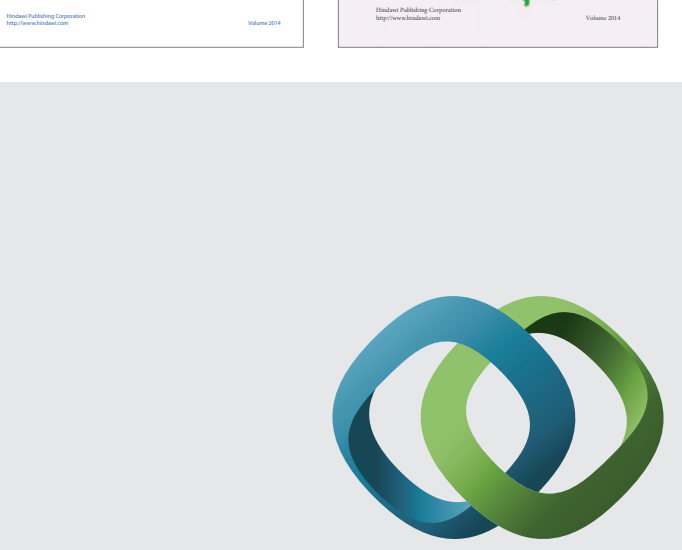

\section{Hindawi}

Submit your manuscripts at

http://www.hindawi.com
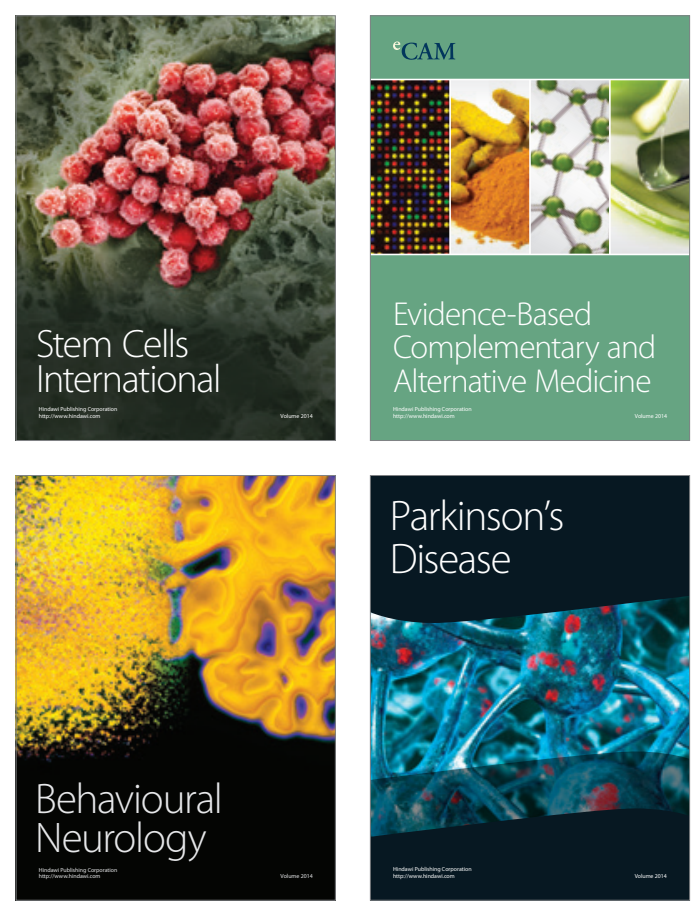

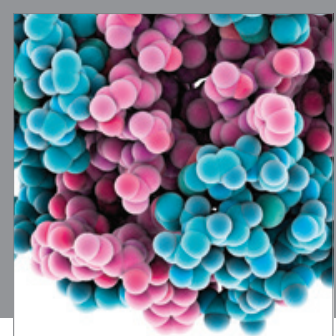

Journal of
Diabetes Research

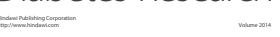

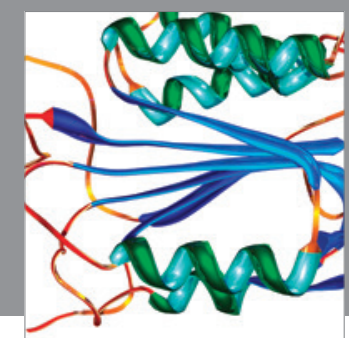

Disease Markers
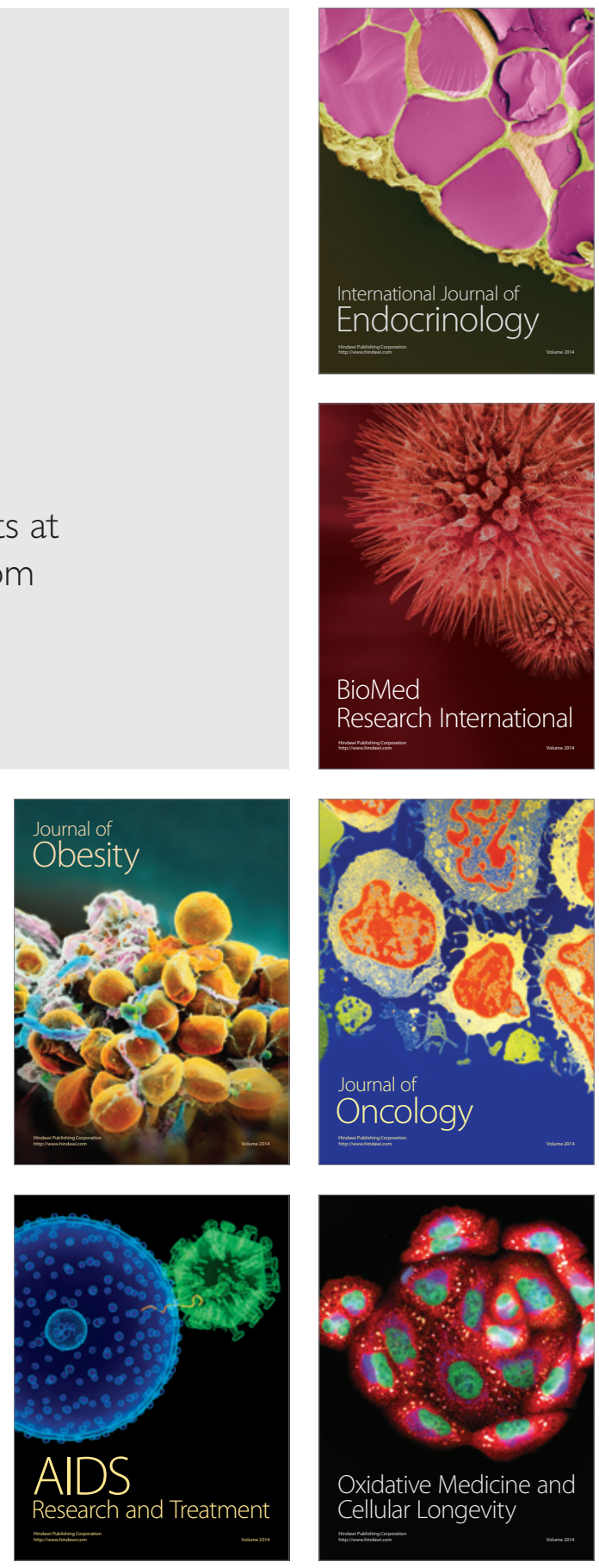